\title{
Albrecht von Haller und Padua
}

Zur Wirkung seines medizinischen Denkens am Ende des 18. Jahrhunderts*

Von Loris Premuda

Zum Medizinstudium ließ sich in Tübingen 1724 als Fünfzehnjähriger Albrecht von Haller (1708-1777) immatrikulieren, der einer der hervorragendsten und entscheidendsten Männer seines Jahrhunderts auf dem mühsamen Weg zu einer wissenschaftlichen Medizin wurde. Obwohl der Ruf der medizinischen Fakultät in Tübingen damals nicht der allerbeste war, hat «doch ... der sechzehnmonatige Aufenthalt Haller sicher nicht geschadet», wie der Medizinhistoriker Henry E. Sigerist (1891-1957) betont. In der Tat: «Das fröhliche Leben und Treiben der Studenten, der Einfluß leichtsinniger veranlagter Kameraden, der Umgang mit den hübschen, 'nicht eben spröden' Tübinger Damen rissen den frühreifen Jüngling, der sich bisher überall wie ein Fremdkörper vorgekommen war, aus seiner Vereinsamung heraus und machten ihn weltoffener. ${ }^{1}$

An der Universität Padua, die schon seit fünf Jahrhunderten bestand, lehrte zur gleichen Zeit bereits seit zehn Jahren mit großem Eifer Giovanni Battista Morgagni (1682-1771), der in mühsamem und methodischem Vorgehen nach und nach wertvolles Material für die Herausgabe seines großen Werkes «De sedibus et causis morborum per anatomen indagatis» sammelte. Seine Arbeit sollte die anatomischen und systematischen Grundlagen der neuen wissenschaftlichen Pathologie schaffen.

Während seines sechzigjährigen Lehramtes in Padua fehlte es dem berühmten Lehrer, trotz Neid und übler Nachrede von seiten seiner Kollegen, nicht an Ehre, Anerkennung und Reichtum. Vor allem konnte Morgagni eine Schule gründen, die sich auf die Richtlinien seines anatomisch-klinischen Denkens stützte. Seine Schüler waren Scarpa (1747-1832), Girardi (1731-1797), Dalla Bona (1712-1786) und Scovolo (gest. 1776), um nur die wichtigsten zu erwähnen. Mit dem Tode Morgagnis kam es in Padua zum Niedergang seines wegweisenden Gedankens: Scarpa und andere Schüler verpflanzten seine Anatomie an andere Orte, und die Schule von Paris eignete sich seine anatomisch-klinische Einstellung an und brachte sie durch Corvisart (1755-1821) und Laënnec (1781-1826) auf einen

* Gastvortrag in der Universität Tübingen auf Einladung des Medizinhistorischen Instituts am 23. April 1975. 
Höhepunkt. Dalla Bona, der Gründer einer klinischen Schule in Padua im Jahre 1765 mit der Bezeichnung «Ad practicam Medicinae in Nosocomio», war dagegen nicht imstande, die anatomisch-klinische Überlieferung seines Lehrers lebendig zu halten und fortzuführen.

Die Gründe für die Unterbrechung der Morgagnischen Tradition in Padua stellen ein größeres geschichtliches Problem dar, als es auf den ersten Blick scheinen könnte. Sie verlangen deshalb eine Untersuchung im einzelnen, der wir uns jetzt zuwenden wollen.

In Padua wurde die Autorität Morgagnis abgelöst durch das große Ansehen, das Albrecht von Haller auch hier gewann. Unsere Aufgabe wird es sein, diesen neuen Hallerschen Einfluß in Padua zu analysieren und zu verstehen. Als Nachfolger auf Morgagnis Lehrstuhl «Ad Anatomen Ordinariam»² wurde Leopoldo Marc'Antonio Caldani (1725-1813) berufen, ein fähiger Mann, der aber die eventuelle Konkurrenz Scarpas unter den Mitbewerbern fürchtete ${ }^{3}$. Caldani kam wie Morgagni aus Bologna. Morgagni war in jener Stadt Schüler von Valsalva (1666-1723) gewesen.

Um es vorwegzunehmen: Caldani wird in erster Linie für die Verbreitung des Hallerschen Denkens in den wissenschaftlichen Kreisen Paduas verantwortlich sein. Er kommt nämlich bereits als überzeugter Hallerianer nach Padua. Und gerade, weil er Hallerianer und deswegen Antitraditionalist war, hatte er schon in Bologna viel Erfolg gehabt; zugleich zog er aber dadurch Neid und Mißgunst auf sich, die ihm das Leben erschwerten ${ }^{4}$. Wir sind im Jahre 1761 : die venezianische Republik, die nach dem Frieden von Passarowitz (1718) und der folgenden Abtretung von Morea immer mehr darauf verzichtete, aktiv in die Orientund Mittelmeerpolitik einzugreifen, geht ihrem verhängnisvollen Untergang entgegen. Das berühmte «Studio» von Padua erlebt in negativer Weise die unglückliche politische Situation. Aber die aktive und einflußreiche Gegenwart von Morgagni und einigen anderen fähigen Lehrkräften wie Dalla Bona, Bonioli (1729-1791) und Dalla Decima (1752-1825) tragen dazu bei, die Tradition lebendigen Interesses an wissenschaftlicher Forschung aufrechtzuerhalten.

Alle Biographen sind sich darin einig, Caldani als einen eifrigen und überzeugten Nachfolger Hallers in der Lehre von der Irritabilität und der Sensibilität zu betrachten. Erich Hintzsche, einer der erfahrensten Kenner des Werkes des Schweizer Arztes, erinnert daran, daß diese Lehre auch der «Anlaß» war, daß Caldani « 1756 briefliche Verbindung mit Haller aufnahm »..$^{5}$ Es ist einleuchtend, daß sich die weltzugewandten Geister nicht mit dem Stahlschen Begriff «Seele» (1708) oder mit dem Begriffe des Nervenfluidums, Triebwerk der Nerven, von Hoffmann (1660-1742) begnügen konnten. Von daher läßt sich die eifrige Zu- 
stimmung Caldanis zu Hallers Lehre verstehen. Außerdem war gerade auf wissenschaftlichem Gebiet in Italien immer eine Bestrebung lebendig gewesen, neue Kräfte zu entdecken. Die fast gleichzeitigen Entdeckungen von Galvani (1737 bis 1789) und von Volta (1745-1827) zeigen das deutlich ${ }^{6}$.

Wie erwähnt, hatte Caldani schon während seines Aufenthalts in Bologna der Hallerschen Anschauung zugestimmt. Haller hatte die erste Mitteilung seiner Forschungen über Irritabilität und Sensibilität in Göttingen am 22. April und am 6. Mai 1752 veröffentlicht ${ }^{\text {? }}$. In den wissenschaftlichen Kreisen Bolognas hatte man sich sogleich mit den Hallerschen Ergebnissen beschäftigt ${ }^{8}$. Caldani konnte seine ersten Bemerkungen in einer brieflichen Arbeit darlegen, die an Haller gerichtet war und in der Akademie des wissenschaftlichen Institutes von Bologna am 25. November 1756 vorgetragen wurde ${ }^{9}$. Das geschah fünf Jahre vor Caldanis Eintritt in den Lehrkörper von Padua. Über die Ersetzung Morgagnis durch Caldani ${ }^{10}$ und über die Verbindung Morgagnis mit Haller ${ }^{11}$ ist viel veröffentlicht worden. Wir beschränken uns darauf, zu unterstreichen, daß es sich zunächst nur um eine «geduldete» Nachfolge handelte. Den Sieg Caldanis hatte «Sua Maestà Anatomica», wie Morgagni genannt wurde, sicher nicht erwünscht. Morgagni hielt durch gut 23 Jahre briefliche Verbindung mit Haller ${ }^{12}$; doch ließ er sich nicht davon abhalten, eine Kritik über seine «Fisiologia» und über Caldanis «Riflessioni fisiologiche» zu schreiben, die uns beide handschriftlich überliefert sind ${ }^{13}$.

Die Feindseligkeiten, denen Caldani bei seiner Lehrtätigkeit begegnete, wurden sicher auch durch einige negative Seiten seines Charakters ausgelöst ${ }^{14}$; vor allem aber beruhten sie auf der überzeugten Zustimmung des Meisters zur Hallerschen Lehre. Es ist sicher, daß zwischen den beiden $W$ issenschaftlern eine warme und tiefe Freundschaft bestand, die vom siebzehnjährigen Altersunterschied der beiden Persönlichkeiten nicht gestört wurde.

Hintzsches Beiträge zur Kenntnis der Beziehungen zwischen Morgagni beziehungsweise Caldani und Haller haben die Kenntnis der Biographien dieser Wissenschaftler bereichert und für die Wissenschaft im Zeitalter der Auf klärung neue Perspektiven eröffnet. Diese ausgezeichneten Beiträge sind wohlbekannt. Wir wollen deshalb nicht die in ihnen durchgeführte Analyse wiederaufnehmen, sondern an die Bewertung der Wirkung gehen, die vom wissenschaftlichen Denken und von der Persönlichkeit Hallers in Padua ausgeübt wurde. Daß das Thema Haller immer lebendig und zeitgemäß ist, zeigt uns das wertvolle Buch von Richard Toellner, einem Schüler von Karl Eduard Rothschuh, «Albrecht von Haller. Über die Einheit im Denken des letzten Universalgelehrten ». ${ }^{15}$

Unsere Untersuchung möchte einerseits den Einfluß des Denkens eines großen 
ausländischen Arztes in einem Universitätszentrum wie Padua darstellen und damit andererseits die Teilnahme italienischer Wissenschaftler an den allgemeinen Tendenzen ihrer Zeit nachweisen, wie Benedetto Croce schon vor fünfzig Jahren hervorhob: «Sprache, Literatur, Gedanken, Sitten und die Männer Italiens selbst nahmen mit ihrer besonderen Begabung und Tüchtigkeit in den Jahrhunderten des politischen Verfalls Italiens noch fast bis zum Anfang der französischen Revolution am sozialen und kulturellen Leben Europas Anteil, einen weitaus größeren Anteil als den, den sie in der folgenden Periode des Risorgimento und der erlangten Unabhängigkeit hatten. ${ }^{16}$

Zu dem Thema, das wir untersuchen möchten, haben wir geeignete Quellen ausgewählt. Geben wir gleich dem berühmten venezianischen Drucker Sebastiano Coletti $^{17}$ das Wort, der im Jahre 1765 die italienische Ausgabe ${ }^{18}$ der "Primae lineae physiologiae» von Haller, die schon 1747 erschienen waren, besorgt hat. Der Drucker möchte sich persönlich an die jungen «Medizinstudenten» wenden; so sehr ist er von dem besonderen Wert des von ihm herausgegebenen Werkes überzeugt. Daraus, meint er, werden sie «den Kern der gesamten theoretischen Medizin erlernen . $^{19}$ «Die wahre Medizin steht nämlich auf zwei Füßen, und zwar der praktischen und der theoretischen Medizin, und ... wer die eine ohne die andere erlernt, hinkt im Ausüben dieser Kunst. » ${ }^{20}$ Nach den Worten Colettis ist das Werk, das er italienisch vorlegt, «ein Auszug der besten physiologischen Lehren, die bis jetzt bekannt sind ... es ist ein wertvolles Produkt eines der gelehrtesten und ausgezeichnetsten Kommentatoren des hervorragenden und nie genügend gefeierten Boerrhave(!): es ist endlich das Werk eines ehrenwerten Philosophen und Arztes, der sein ganzes Leben lang nichts anderes vor Augen hatte als das Suchen nach Wahrheit, der sich weder fürchtete noch schämte, sich zu widersprechen oder seine Ansicht zu wechseln, wenn wiederholte und sichere Erfahrungen ihn überzeugten, anders zu denken als andere, wenn auch sehr berühmte Männer. ${ }^{21}$

Aus den Worten des Druckers, die auch auf die Notwendigkeit hinweisen, ein lateinisch geschriebenes Werk in die Volkssprache zu übersetzen und das zu rechtfertigen, erkennt man die neue Funktion einer «Philosophie», die von der aufklärerischen Doktrin beeinflußt ist. Sowohl die Philosophie wie auch die Wissenschaft müssen aus dem engen Kreis der Gelehrten hinaustreten und in alle sozialen Schichten eindringen. Indem man ein Werk verbreitet und dem Volke zugänglich macht, kann zwar die traditionelle Würde des wissenschaftlichen Werkes leiden; doch vermag dieser Vorgang den wissenschaftlichen Gedanken lebendiger und unbefangener zu erhalten. Auch ein Drucker hat am Bau einer neuen Kultur und einer neuen Lebens- und Weltanschauung Anteil, vor allem, wenn 
ein solches Werk durch neue und frische Kenntnis der menschlichen Physiologie erarbeitet wurde, wie sie der berühmte Haller darlegte, Haller, dem es von vielen Gesichtspunkten aus gelang, «modern» zu erscheinen und zu sein und der damit ein sogar von Kant hochgeachteter Lehrer wurde.

Daß vor allem Padua Hallers Werk mit Ungeduld erwartete, geht klar aus einer Prüfung der «Rotuli» des Archivs der Universität hervor. Caldani, der den Lehrstuhl der «Theorica Ordinaria Medicina» innehatte und seit 1769 alternierend Physiologie und Pathologie lehrte, verlangte und erhielt 1771 von den Reformern der Universität die Erlaubnis, «primas lineas physiologiae Halleri» zu erläutern ${ }^{22}$, und im gleichen Jahr, in dem auch Morgagni stirbt, wird auch Scovoli, sein Schüler, verlangen, in seinen Vorlesungen «de morbis particularibus» «nonnullas hypotheses Halleri» ${ }^{23}$ darlegen und anwenden zu dürfen. Als Caldani am 21.Januar 1764 an Giovanni Bianchi (1695-1775), einen der schärfsten Köpfe der Zeit, schrieb, bezeichnete er Haller als einen « wahren gemeinsamen Freund ». ${ }^{24}$ Über Kupferstiche, die alle Details des Innenohrs «mit größter Genauigkeit und Naturtreue» darstellen sollten, «damit auch der unerfahrene Leser sich eine klare und deutliche Vorstellung von der Zusammensetzung des Organs sowohl beim neunmonatigen Kleinkind wie auch beim erwachsenen Menschen machen kann », ${ }^{25}$ berichtete er in diesem Zusammenhang: «Die Präparate des Foetus waren fast alle vollendet, ... besonders, was die alten Knochen betrifft, und damit sie mit größter Genauigkeit gestochen werden könnten, hatte ich sie Herrn Haller gesandt, damit er sie mir von seinem berühmten Stecher stechen lassen möge, da in Venedig die Stecher leider keine große Gewandtheit in solchen Sachen haben und noch weniger die Maler. Ich war nämlich überaus großen Schwierigkeiten begegnet, sie zeichnen zu lassen, und es war mir erst gelungen, nachdem ich dem Maler die genaue Anatomie dieser Teile beigebracht hatte. ${ }^{26}$ Bei dem erwähnten Kupferstecher kann es sich weder um J.P.Kaltenhofer (gest. 1777) noch um G.D.Heumann (1691-1759), die beiden berühmtesten Mitarbeiter Hallers, handeln, denn der erste starb viel später, der andere fünf Jahre vor dem von Caldani geäußerten Wunsch an Haller. Von Caldani erfahren wir aber, daß der Stecher leider etwa zwei Monate vor seiner Zusendung der Zeichnungen gestorben sei ${ }^{27}$. Dieses Ereignis ist anscheinend nicht von großer Bedeutung; es hilft uns aber, die Hochachtung vor der Equipe mit Haller an der Spitze unter den Wissenschaftlern des venetischen Gebiets zu erkennen.

Untersuchen wir nun von einem anderen Gesichtspunkt aus die Wirkung des medizinischen Denkens von Haller. Das führt uns zu dem besten Biographen Caldanis, seinem Neffen Floriano Caldani (1772-1836). Dieser promovierte 1792 in Padua, und diese Promotion war eine günstige Gelegenheit, dem jungen Arzt 
als Gratulationsgeschenk nach damaligem Brauch eine kleine Abhandlung zu überreichen. Der Herausgeber des erfolgreichen Büchleins war Francesco Fanzago (1764-1836), Schüler des Leopoldo Marc'Antonio Caldani und zu jener Zeit erst achtundzwanzigjährig ${ }^{28}$. Er benutzte offenbar diesen Anlaß, um sich die Sympathien des einflußreichen Onkels von Floriano zu erwerben. An Caladani ist denn auch eine vier Seiten lange Widmung gerichtet, in der er als «liebevollster Onkel des Promovierten » bezeichnet wird. Was enthielt nun das kleine Büchlein als thematischen Kern? Nichts anderes als die volkssprachliche Übersetzung eines Aufsatzes von Haller über den Ruhm ${ }^{29}$. Das ist ein beziehungsreicher und lebendiger Beweis für die Berühmtheit des großen Physiologen in Italien. Zugleich zeugt es von der Gewißheit des Herausgebers, mit der Auswahl eines Werkes, das vom Empfänger der Widmung mit Freuden aufgenommen wird, weil es von seinem hervorragenden und verehrten Freund geschrieben wurde, ins Schwarze zu treffen. Im Widmungsbrief hebt Fanzago die Gefühle der gegenseitigen Freundschaft zwischen den beiden Persönlichkeiten hervor und erwähnt die Widmung einiger Werke Hallers an Caldani ${ }^{30}$. Abschließend schreibt er: «Jene poetische Abhandlung über den Ruhm, die er [Haller] an seinen lieben Freund Giller bei der Promotion zum Doktor richtete, lebe an diesem Tag der Freude in unserer Mundart wieder auf, und der Name Gillers verwandle sich in jenen eures Floriano. ${ }^{31}$

Von diesem Floriano Caldani erhalten wir in der Monographie über das Leben und die Werke seines Onkels ${ }^{32}$ weitere Nachrichten über Hallers Stellung und Bedeutung in der kulturellen Situation von Padua. Er bemerkt, welch ausschlaggebende Wirkung für die Ausbildung des Onkels seine Bekanntschaft mit den «Institutiones Medicae» von Hallers Lehrer Boerhaave hatte und wie Caldani daraufhin sich nicht mehr so sehr an den alten klassischen Autoren von Aristoteles und Avicenna bis zu Vesal und Eustachi orientiert habe als vielmehr stets auf der Suche nach Neuem «durch wiederholte anatomische Sektionen » ${ }^{33}$ gewesen sei. Diese Neuorientierung war wahrscheinlich ausschlaggebend für das Zusammentreffen der wissenschaftlichen Interessen von Caldani und Haller. Floriano weist auch darauf hin, daß die Freundschaft mit Haller für die Berufungen Caldanis durch deutsche Universitäten und durch Fürsten ${ }^{34}$ wohl eine wesentliche Rolle gespielt habe, und betont nochmals, wie wichtig es gewesen sei, Hallers «Primae lineae physiologiae» als Grundlage für den Unterricht in Padua zu wählen ${ }^{35}$.

Caldani hatte freundschaftliche Briefbeziehungen mit Bonnet (1720-1793) in Genf, mit Albinus (1697-1770) und Sandifort (1740-1819) in Leiden, mit Blumenbach (1752-1840) in Göttingen, der ihm die Dissertation «De oculis leucaethio- 
pum et iridis motu» widmete; auch mit van Swieten (1700-1772), mit Frank (1745-1821) und mit vielen anderen ${ }^{36}$ hielt er Verbindung. Aber in erster Linie betont der Neffe die «vertrauliche Freundschaft, zu der sich der große Haller, der Mann des Jahrhunderts » ${ }^{37}$ öffentlich bekannte. Auch die weniger positiven Aspekte des Hallerschen Denkens wie die Aufnahme «der berühmten Hypothese der animalischen Spiritus» «in seine große Physiologie» werden mit Wohlwollen beurteilt, hatte doch jene Vermutung letztlich Caldani zu seinen experimentellen Forschungen für eine mögliche Erhärtung dieser Hypothese angespornt, die dann aber im Gegenteil zu ihrer Widerlegung führten ${ }^{38}$.

An anderer Stelle ${ }^{39}$ zitiert Floriano als hervorragendste Zeugen für die Größe des «Studio» von Padua den «gelehrten Haller» und den Franzosen Portal (um 1650 bis 1703), da sie als Geschichtsschreiber bestätigen, daß es ein «rühmlicher Ausweis» für einen Arzt oder Anatomen sei, an jener Universität studiert zu haben ${ }^{40}$. Anderswo lobt er das unbestreitbare Urteil «des großen Haller», wenn dieser die führende Rolle Harveys (1578-1657), des Schülers von Fabrici d'Acquapendente in Padua, für die Wiedergeburt der englischen Anatomie hervorhebt ${ }^{41}$.

An der Macht der «Persönlichkeit» Hallers und des Hallerschen Denkens in der Universitätswelt und in der ärztlichen Welt von Padua können keine Zweifel bestehen. Aufgrund der einflußreichen und überzeugten Parteinahme Caldanis hatte sich Hallers Denken auf der gelehrten Ebene in weitem Umfang durchgesetzt. Aber noch mächtiger ist der Erfolg eines neuen Denkens, wenn dieses die Schranken einer begrenzten Disziplin durchbricht und sich unter den Gelehrten und Förderern anderer Disziplinen verbreitet, so daß der Name eines Wissenschaftlers in allen Schichten und in vielen Wissensbereichen bekannt wird. Das ist mit Sicherheit bei Haller im kulturellen Raum von Padua, ja im ganzen venetischen Bereich der Fall gewesen. Eindrucksvollster Beweis dafür ist eine heute fast vergessene Sammlung ${ }^{42}$ von Prosaschriften und Gedichten, die 1780 in Padua «beim Tode des großen Albrecht von Haller » ${ }^{43}$ veröffentlicht wurde.

Diejenigen, die diese Anthologie zusammenstellten, haben verschiedenste kulturelle Wurzeln und unterschiedliche Anliegen. Das 14 seitige Vorwort in Prosa wurde von Leopoldo Marc'Antonio Caldani verfaßt. Er richtete es an «Seine Exzellenz den hochverehrten und gelehrten Mylord Graf von Bute» (1713-1792), der ein großer Freund des Verstorbenen und zugleich Politiker und Minister Georgs III. (1738-1820), des Königs von Hannover und England, war ${ }^{44}$. Auf den übrigen 100 Seiten finden wir Beiträge in Prosa von Francesco Albergati Capacelli (1728-1804) und in Versform von Pellegrino Gaudenzi (1749-?), von Girolamo Ruggia (1748-1822), von Giuseppe Gennari (1721-1800) und von 
Antonio Gardini (1742-1807). Wer sind diese Autoren? Es sei gleich gesagt: es sind keine Männer, die in der Geschichte der italienischen Kultur eine hervorragende Rolle spielten. Zu ihrer Zeit aber haben sie durchaus Bedeutung und Einfluß gehabt.

Albergati Capacelli, von Bologna gebürtig, war ein enger Freund Voltaires, Goldonis und Alfieris. Er war ein begeisterter Theaterfreund und schrieb selber Komödien. In seinen Werken, die nicht immer erfolgreich waren, findet man satirische und moralisierende Anspielungen der Gesellschaft seiner Zeit gegenüber, zu denen er von Goldoni oder vielleicht sogar von Diderot angeregt wurde ${ }^{45}$. Gaudenzi, ein Schüler von Melchiore Cesarotti (1730-1808), hatte den Lehrstuhl für Griechisch und Hebräisch an der Universität Padua inne und war Sekretär der «Accademia patavina di scienze, lettere ed arti». Er widmete sich Studien in der Geometrie und der analytischen Mathematik, war mit Physik und Mechanik vertraut, versuchte sich aber auch in der Dichtkunst ${ }^{46}$. Ruggia schrieb Gedichte, die meist in Parma und Bologna gedruckt wurden ${ }^{47}$, während Gennari ein Historiker war, der unter anderem die «Annali padovani» (Bassano 1804) ${ }^{48}$ verfaßte. Gardini schließlich gehörte zur Gruppe jener Männer, die sich mit Ästhetik beschäftigten und sich in Padua um Cesarotti ${ }^{49}$ sammelten.

Sehen wir kurz den Inhalt der jeweiligen Beiträge durch. Im Widmungsbrief rühmt Caldani zunächst Hallers Unparteilichkeit und Liebe zur Wahrheit, durch die er «den italienischen Autoren jene Gerechtigkeit widerfahren ließ, die ihnen mit Recht zugestanden werden $\mathrm{mu} ß{ }^{50}$ Caldanis Gedanken verraten einen neuen Sinn für das nationale Selbstbewußtsein, das seit langem unter den Italienern erloschen schien. Das ist wohl eine Folge der Ereignisse nach dem Frieden von Aachen, der den langwierigen Kämpfen zwischen den fremden Völkern um die Vorherrschaft in Italien ein Ende setzte und den Druck Spaniens auf Italien beseitigte. Caldani betont, daß es nicht leicht sei, Haller zu würdigen, einen Schriftsteller, der sich in der Dichtung wie in der Botanik, in der Physiologie, in der Anatomie, in der Medizin und in der Politik ${ }^{51}$ ausgezeichnet habe, und er erwähnt als Zeichen seines Ruhms, daß dem, der in Bern nach Haller fragte, erwidert wurde: «Meinen Sie den Großen ? „52 Er bedauert, Haller wegen unglücklicher Umstände nicht persönlich kennengelernt zu haben, und versichert dem Grafen von Bute, daß niemand besser als er, der mit Haller gut bekannt war, beurteilen könne, daß der Professor von Göttingen wirklich als «gro $\beta{ }^{53}$ zu betrachten sei.

Albergati Capacelli bringt eine Biographie Hallers auf 44 Seiten ${ }^{54}$. Eine aufklärerische Haltung durchdringt die ganze Abhandlung und wird schon am Anfang offenbar: Cäsar, Alexander und Karl von Schweden würden heute «nichts 
anderes als Abscheu und Schauder ${ }^{55}$ erwecken. Friede und Bildung seien allgemeiner Wunsch: nur der friedliche und gelehrte Mensch verdiene freundliche Ehrungen im Leben und Trauer beim Tode. Verständlich sei der Schmerz Frankreichs, Italiens, Deutschlands über den Verlust eines Voltaire, eines Zanotti, eines Haller ${ }^{56}$.

Albergati verfolgt dann im einzelnen die Lebensereignisse des großen Physiologen. Er sieht ihn als enfant prodige, das sich «mit wunderbarem Flug über den gemeinen Stand erhebt». ${ }^{57}$ Bei Haller sind mehr noch als die breiten philologischen Kenntnisse die Verbindung von «tiefem Geist» und «reger Vorstellungskraft ${ }^{58} \mathrm{zu}$ bewundern. Wichtige Etappen seiner wissenschaftlichen Bildung sind Leiden und Basel: auf der einen Seite Boerhaave und Albinus, auf der anderen Johann I Bernoulli, ein hervorragender Mathematiker. In seiner Vorliebe für Bildungsreisen ist eine Berührung mit den altgriechischen Philosophen zu erkennen, die durch jedes Land wanderten, in dem sie «lehrreiche Kenntnisse finden zu können ${ }^{59}$ glaubten. Breit und aufbauend waren seine Arbeiten für Botanik und Anatomie, «und hoch und glänzend war wohl der Preis für seine mit so großem Elan durchgeführten anatomischen Versuche, ... da er durch diese Versuche nützliche Klarheit dort schuf und verbreitete, wo vorher Finsternis und Zweifel dem menschlichen Verstand im Wege standen.» ${ }^{60}$ Albergati verweist auch auf die praktischen Folgen von Hallers Entdeckung der Irritabilität für Medizin und Chirurgie ${ }^{61}$.

In Italien war nach Albergati Bologna der Mittelpunkt, von wo die Hallersche Lehre ausstrahlte. Padua war die Festung, von der aus seine Anschauungen «durch den ersten kraftvollen und hochangesehenen Anhänger Hallers» ${ }^{62}$, also Caldani, verteidigt wurden. Aber, bemerkt Albergati, nachdem die Widerstände und Schwierigkeiten beseitigt waren, «verbreitete sich das Hallersche System an den bedeutendsten Universitäten Europas und gründete dort eine ewige Herrschaft». ${ }^{63}$ Auffallend sind die Ähnlichkeiten, die Albergati zwischen Zanotti, Voltaire und Haller feststellt. Alle drei «schienen als Dichter geboren zu sein " ${ }^{64}$; alle drei erwarben später großen Ruhm auf anderen Gebieten; alle drei wünschten in dem Lande, wo sie geboren wurden, ihr Leben zu beenden: in Bologna, in Paris, in Bern ${ }^{65}$.

Damit sind wir bei den vier Beiträgen in Versform angelangt. Wir wollen keine Übersetzung geben, sondern uns auf einige Hinweise beschränken. Der erste Beitrag stammt von Pellegrino Gaudenzi, «einem Schüler der Akademie von Padua», und trägt den Titel «Die Trauer der Alpen». ${ }^{66}$ Es spielt damit auf jenes Gedicht an, in dem Haller schon 1728/29 die "Alpen» besungen hatte, jenes Gedicht, das, wie Tonelli feststellt, «unter den zahlreichen Gedichten 
Hallers sicher die größten Erörterungen in der Kritik entfacht hat ». ${ }^{67}$ Gaudenzis Trauergedicht besteht aus 254 reimlosen elfsilbigen Versen mit einem Zwischenspiel und einem Kanzonettenfinale. Es weist die Züge arkadischer Lyrik auf, die oft erkünstelt wirkt und nur selten die Töne echten Gefühls zeigt. Und doch findet man darin auch jenen melancholischen Ton englischer Herkunft. So in dem Bild:

«Erloschen ist der Sänger der Alpen, und erloschen sind /

auf dem Hügel die süßen Weisen und die Harmonie, /

die das Herz nährte ... » ${ }^{68}$

Gaudenzis Poesie läßt sich mit der von Jacopo Vittorelli (1749-1835), einem zeitgenössischen venetischen Dichter, vergleichen.

Der «Gesang des Herrn Abt Girolamo Ruggia » besteht aus 113 Elfsilbern und enthält häufige Anspielungen auf die klassische Mythologie. Wir erkennen seine polemisch ausgedrückte Abneigung gegen den Secentismus und seinen Willen zu einer Reform der Lyrik in klassizistischem Sinn.

«Aller (!), du liegst, und von der eisernen Nacht /

des Erebos ausgelöscht, drücke nun harte Stille auf deine Lippen. » ${ }^{69}$

Gennari benützt ebenfalls die reimlosen Elfsilber (insgesamt 163) in seinem Poem, das sich an den Ritter Clementino Vannetti (1754-1795) wendet:

«Erloschen ist Helvetiens Licht, /

vielmehr das Licht Europas. Blind und leidend /

weint die Medizin .... ${ }^{70}$

Im ganzen Gedicht herrscht die Gelehrsamkeit des 18. Jahrhunderts vor, eingebettet in einen Rahmen ehrwürdiger Klassik. Häufig finden wir spezifische wissenschaftliche Anspielungen:

«Wenn mein Caldani in den sterblichen Schlamm, /

der uns umgibt, die Hände eintaucht, /

und als durch und durch ehrwürdiger Anatom /

die irritable Faser oder die sensiblen Nerven, /

Venen und Arterien, Knochen und Membranen zeigt, /

lobt er oft des Albinus und Morgagnis und/

des gelehrten Haller schöne Entdeckungen. ${ }^{71}$

Auch Gardini bedient sich der reimlosen Elfsilber (im ganzen 112) und teilt sie in Stanzen ein. Das kleine Gedicht trägt die Züge neuklassischer Dichtung und ist durch und durch eine Verherrlichung der berühmten Persönlichkeit Hallers. 
Wie sind uns bewußt, mit diesem Beitrag zur Aufnahme Hallers in Italien nicht so sehr ärztlich-biologische Probleme beleuchtet zu haben; wohl aber glauben wir, eine kleine Lücke in der Kenntnis der verwickelten Lebensgeschichte dieses hervorragenden Universalgelehrten, seiner Beziehungen und Einflüsse gefüllt zu haben. Was die «Universalität» von Hallers Werk betrifft, das nicht nur wissenschaftlich, dichterisch, geschichtlich und politisch, sondern wahrscheinlich noch mehr war, so möchten wir abschließend daran erinnern, daß Caldani an jenem Abend des 24. Dezember 1813, als er sich aufs Lager legte, von dem er nicht mehr aufstehen sollte, «jenes lange Stück der Hallerschen Physiologie durchlesen wollte, wo von der Seele gesprochen wird ». ${ }^{72}$ Es will uns dies wie die höchste Anerkennung der Größe eines Wissenschaftlers erscheinen.

\section{Anmerkungen}

${ }^{1}$ H.Sigerist, Große Ärzte, München 1932, S. 141.

${ }^{2}$ Vgl. dazu: G. Bilancioni, La morte e la successione del Morgagni secondo un anatomico contemporaneo. In: Veteris vestigia flammae, Roma 1922, S. 465-502. Dieselbe Arbeit wurde zuvor publiziert in: Rivista di storia delle scienze mediche e naturali 6 (1915) 487-509.

${ }^{3}$ Vgl. G. Favaro, Antonio Scarpa i i Caldani. In: Atti R. Ist.Ven.di S.S.L.L.A.A., Tomo 91, Parte II (1931-32), S. 23-33.

4 Vgl. dazu P. Capparoni, Leopoldo Caldani. In: Profili bio-bibliografici di medici e naturalisti celebri italiani dal sec. XV al sec. XVIII, Vol. 2, Roma 1928, S. 92 f.; F.Puccinotti, Storia della Medicina, Vol.3, Prato 1866, S. 347-349; E. Hintzsche, Leopoldo Marcantonio Caldani. In: Dictionnary of Scientif. Biogr. (Ed. Gillispie), Vol. 3, New York 1971, S.15f.; Opere del Conte Algarotti, Edizione novissima, Tom. 10, Venezia 1794, S. 49-63; F.Zanotti, De quibusdam animalium partibus, an sensu sint compotes, et unde irritabilitas. In: De Bononiensi Scientiarum et Artium Instituto atque Academia Commentarii, Tom.4, Bologna 1757, S. 48-57.

5 Albrecht von Haller-Marc Antonio Caldani, Briefwechsel 1756-1776, hrsg. und erläutert von Erich Hintzsche, Bern/Stuttgart 1966, S.224.

${ }^{6}$ Man beachte auch das Interesse Caldanis an der exakten Bestimmung des Einflusses eines elektrischen Reizes auf die Herztätigkeit. Vgl. dazu M.Neuburger, Die historische Entwicklung der experimentellen Gehirn- und Rückenmarksphysiologie vor Flourens, Stuttgart 1897, S. 154-156.

7 A. von Haller, De partibus corporis humani sensilibus et irritabilibus. In: Commentarii Soc. Reg. Sci. Gottingensis 1752 (2), Göttingen 1753, S.114-158.

8 Vgl. V.Busacchi, Tommaso Laghi bolognese (1709-1764). Saggio bio-bibliografico. In: Atti Mem. Accad. Stor. Arte San. 1 (1935) 11-18. S. außerdem De Bononiensi Scientiarum ... (s. Anm. 4): Thomae Laghii, De insensibilitate, atque irritabilitate Halleriana, S. 208. 
${ }^{9}$ L.M. A. Caldani, Sull'insensitività, ed irritabilità di alcune parti degli animali, Bologna 1757.

10 Op. cit. (s. Anm. 2), S. 467 f., und G. Ongaro, Leopoldo Marc'Antonio Caldani e Albrecht von Haller. In: Atti del XXIII Congresso Nazionale di Storia della Medicina (Modena 22-24 settembre 1967), Roma 1967, S. 618-623.

11 A.v. Haller und G. Morgagni, Briefwechsel 1745-1768, hrsg. und erläutert von E. Hintzsche, Bern/Stuttgart 1964.

12 Op. cit. (s. Anm. 5).

${ }^{13}$ G. Bilancioni, op. cit. (s. Anm.2), S.491 f. und 501;G. Ongaro, op. cit.(s. Anm.10), 622 f.; D. Giordano, Morgagni, Torino 1941, S. 260.

14 Op. cit. (s. Anm. 5), S. 227.

15 R.Toellner, Albrecht von Haller. Über die Einheit im Denken des letzten Universalgelehrten. Wiesbaden 1971 (= Sudhoffs Archiv, Beihefte, H.10).

16 B. Croce, Uomini e cose della vecchia Italia, Serie seconda, Bari 1927, S. 1.

17 Die Coletti (oder: Coleti) waren eine bedeutende Drucker- und Buchhändlerfamilie in Venedig. Sebastiano veröffentlichte zusammen mit seinem Bruder Nicolò eine neue Ausgabe von Ughellis Italia sacra und sammelte den ersten Kern der Bibliothek. Vgl. dazu C.Frati, Dizion. bio-bibliogr. di bibliotecari e bibliofili italiani, Firenze 1952, S. 274-275; G.A.Moschini, Della letteratura veneziana del secolo XVIII, Venezia 1806, Tom. 2, passim; Fortsetzung und Ergänzungen zu Ch. G. Jöchers allgemeinem Gelehrten-Lexicon ... von J. Ch. Adelung, Bd. 2, Hildesheim 1960 (Nachdruck der Ausgabe Leipzig 1787), S. 410 .

${ }^{18}$ I primi lineamenti di Fisiologia del Signor Alberto Hallero dalla lingua latina trasportati nell'Italiana da un Professore di Medicina, Venezia 1765.

19 Ib., S. III.

20 Ib., S. IV.

21 Ib., S. V.

${ }^{22}$ B. Bertolaso, Ricerche d'archivio su alcuni aspetti dell'insegnamento medico presso l'Università di Padova nel Sette- ed Ottocento. In: Acta Medicae Historiae Patavina 5 (1958-59) 8.

${ }^{23}$ Ib., S. 8-9.

${ }^{24}$ G. Bilancioni, Per la storia dell'anatomia dell'orecchio - Lettere inedite di Domenico Cotugno e di Leopoldo Marcantonio Caldani. In : Sulle rive del Lete, Roma 1930, S. 186. Die noch nicht zusammengefaßte Arbeit mit dem Titel «Lettere inedite di Leopoldo Marcantonio Caldani » erschien bereits in: Arch. Ital. Rinol. Laring. 26 (1915) 118-132 und 234-257.

25 Ib., S. $186 \mathrm{f}$.

26 Ib., S. 187.

27 Ib.; über die Stecher, die für Haller arbeiteten, vgl. auch L. Premuda, Storia dell'iconografia anatomica, Milano 1957, S. 178-182.

${ }^{28}$ Fanzago war auch Schüler von Gallini (1756-1836) und von Dalla Bona und später Freund von Frank (1745-1821). Im Jahre 1817 wurde er auf den Lehrstuhl «Medicina forense e polizia medica» in Padua berufen.

${ }^{29}$ La Gloria. Componimento del Signor Haller volgarizzato in occasione che prende 
la laurea in filosofia, e medicina il Signor Floriano Caldani, a cura di F. Fanzago, Padova 1792.

30 Op. cit. (s. Anm. 29), S. IV.

31 Ib., S. VI.

${ }^{32}$ Memorie intorno alla vita ed alle opere di Leopoldo Marco Antonio Caldani scritte da Floriano Caldani, Modena 1822. (Das Buch ist gewidmet «A Sua Altezza Reale la Serenissima Maria Beatrice Ricciarda d'Este Archiduchessa d'Austria, Duchessa di Massa e Carrara, ecc.»)

${ }^{33}$ Ib., S. VI.

${ }^{34}$ Ib., S. XXVI-XXVII.

${ }^{35}$ Ib., S. XXXII-XXXIII.

${ }^{36}$ Ib., S. LXXI.

37 Ib.

${ }^{38}$ Ib., S. XXXVI-XXXVII.

${ }^{39}$ Discorso inaugurale letto nella grand'Aula dell'I.R. Università di Padova per l'apertura di tutti gli studii nel giorno XXV di novembre MDCCXXVII da Floriano Caldani professore di Anatomia e d'Introduzione allo studio medicochirurgico, Padova 1828.

40 Ib., S. 23.

${ }^{41} \mathrm{Ib} .$, S. 27.

42 Erich Hintzsche mit seiner besonderen Kenntnis der Haller-Literatur hat mich darin bestätigt, wofür ich ihm herzlich danke. Hinweise in Form bloßer Literaturzitate finden sich in der schon erwähnten Arbeit von G. Ongaro (Sonderdruck S. 26) und im Literaturverzeichnis der Monographie von Toellner (s. Anm. 15), S. 206, Nr. 85.

43 In morte del Grande Alberto di Haller Signore in Goumoens le Jux, et in Eclagmens del Consiglio Sovrano di Berna etc., Padova 1780.

${ }^{44}$ Zum Conte di Bute vgl. G. M. Trevelyan, Storia dell'Inghilterra nel secolo XIX (italienische Übersetzung von G. Martini und E. Panicieri), 3. Aufl., Milano 1967, S. 641 .

${ }^{45} \mathrm{Zu}$ Albergati Capacelli vgl. E. Masi, La vita, i tempi e gli amici di F.A.C., Bologna 1878; A. Butti, Spigolatura d'archivio intorno a F.A. In: Giorn. Stor. d. Lett. Ital. 45 (1905) 175 f.

46 Opere di Pellegrino Gaudenzi, forlivese, Venezia 1811, S. 46.

${ }^{47}$ Von ihm ist wenig bekannt. Er schrieb 2 Bände Poesie (Parma 1806) und La coltura del cuore (Bologna 1809).

${ }^{48}$ Siehe dazu G. Vedova, Biografie degli Scrittori Padovani, Padova 1836, S. 447 bis 456; A. Lombardi, Storia della lett. ital. nel sec. XVIII, Modena 1827-30.

${ }^{49} \mathrm{Zu}$ Gardini vgl. Cenni biografici degli Accademici defunti, a cura di G. Barbieri, in: Nuovi Saggi della Cesarea Regia Accademia di Scienze, Lettere e Arti di Padova, Padova 1817, S. XXX-XXXI.

50 Op. cit. (s. Anm. 43), S. IV.

${ }^{51}$ Ib., S. VI.

${ }^{52}$ Ib., S. IX.

${ }^{53}$ Ib., S. IX-X. 
${ }^{54}$ In morte del celeberrimo Signor Alberto Haller Ragionamento del Signor Marchese Francesco Albergati Capacelli. In: op. cit. (s. Anm. 43), S. 17-63.

55 Ib., S. 20.

56 Ib., S. 21.

57 Ib., S. 27.

${ }^{58}$ Ib., S. 30.

${ }^{59}$ Ib., S. 34 .

60 Ib., S. 44.

${ }^{61}$ Ib., S. 45.

$62 \mathrm{Ib} .$, S. $46 \mathrm{f}$.

63 Ib., S. 48.

64 Ib., S. 57.

65 Ib., S. 57-59.

${ }^{66}$ Ib., S. 65-84.

${ }^{67}$ G. Tonelli, Poesia e pensiero in Albrecht von Haller, Torino 1961, S. 1.

${ }^{68}$ Op. cit. (s. Anm. 43), S. 71.

${ }^{69}$ Canto del Signor Abate Girolamo Ruggia. In: op. cit. (s. Anm. 43), S. 85-90.

70 All'ornatissimo Signor Cavaliere Clementino Vannetti Giuseppe Gennari Socio dell'Accademia di Padova. In: op. cit. (s. Anm. 43), S. 91-99 (hier: S. 91).

${ }^{71}$ Ib., S. 93.

72 Del Signor Abate Antonio Gardin Accademico di Padova, e Precettore delle Scuole Pubbliche, Stanze. In: op. cit. (s. Anm. 43), S. 100-109.

${ }^{73}$ Op. cit. (s. Anm. 32), S. LXXIX.

\section{Summary}

Albrecht von Haller had a correspondence with two great professors of medicine at Padua: Giovanni Battista Morgagni and Leopoldo Marc'Antonio Caldani. While Morgagni was sceptical against Haller's new neurophysiological views, Caldani was their most ardent defender. Documents are quoted, proving the high esteem which Haller enjoyed in Italy. The publisher Sebastiano Coletti printed at Venice, in 1765, an Italian version of Haller's "Primae lineae physiologiae". Caldani's pupil Francesco Fanzago translated Haller's "Essay on the glory" and published it on the occasion of the promotion of Caldani's nephew Floriano. This Floriano has later written a biography of his uncle, where he mentions that Caldani has used Haller's "Primae lineae" for the preparation of his lectures. A proof for the reputation, which Haller had reached in Italy, is a collection of some writings in prose and in verses. They appeared at Padua in 1780 in honour of Haller after his death.

Prof. Dott. Loris Premuda

Istituto di Storia della Medicina

dell'Università di Padova

I-35 100 Padova

Via G. Falloppia, 16 